\title{
Transplantation of Renal Allograft After Removal of Renal Cell Carcinoma: Case Report and Review of the Literature
}

\author{
Anthony P. Tran, ${ }^{1 *}$ Paulo N. Martins, ${ }^{1 *}$ Zachary G. Papazian, ${ }^{5}$ Vijay K. Vanguri, ${ }^{2}$ \\ Babak Movahedi, ${ }^{1}$ Pang-Yen Fan, ${ }^{3}$ Kenneth A. Bodziak, ${ }^{3}$ Jennifer K. Yates, ${ }^{4}$ \\ Mitchell H. Sokoloff, Adel Bozorgzadeh ${ }^{1}$
}

\begin{abstract}
With the rising incidence of end-stage renal disease in the United States, patients needing renal transplants are waiting longer for increasingly scarce grafts. Formerly, the general practice was to avoid organs with tumors for transplant because of the risk of malignancy transmission to the recipient. However, with comprehensive donor selection and a small-sized primary tumor, the positive outcomes of transplant outweigh the risks of transmission after a partial nephrectomy. In our case, a 31-year-old woman, the daughter of the recipient, underwent a laparoscopic nephrectomy with an existing 8-mm tumor later confirmed as renal cell carcinoma. An ex vivo tumor enucleation was performed before the allograft was transplanted into the 69-year-old patient with endstage renal disease. At last follow-up, graft function has remained excellent with no evidence of local recurrence or metastasis in both the donor and recipient. Here, we describe our case and perform a literature review on the incidence and management of renal allografts with incidentally detected renal cell carcinoma during transplant.
\end{abstract}

Key words: Kidney transplantation, Kidney masses, Living donor

\footnotetext{
From the ${ }^{1}$ Department of Surgery, Division of Organ Transplantation, the ${ }^{2}$ Department of Pathology, the ${ }^{3}$ Department of Medicine, Division of Renal Medicine/Nephrology, and the ${ }^{4}$ Department of Urology, University of Massachusetts Medical School and UMass Memorial Medical Center, Worcester, Massachusetts, USA; and the 5 Tufts University, Medford, Massachusetts, USA

Acknowledgements: The authors have no sources of funding for this study and have no conflicts of interest to declare.

*Anthony P. Tran and Paulo N. Martins contributed equally to this work.

Corresponding author: Adel Bozorgzadeh, Department of Surgery, Division of Organ

Transplantation, UMass Memorial Medical Center, University Campus, 55 Lake Avenue North

Worcester, MA 01655, USA

Phone: +1 5083342023 E-mail: Adel.Bozorgzadeh@umassmemorial.org
}

Experimental and Clinical Transplantation (2019)
Introduction

Despite the stable number of patients with end-stage renal disease (ESRD), with 117162 new cases in 2013 in the United States, the prevalence of patients receiving dialysis as treatment for ESRD continues to increase. ${ }^{1}$ As the transplant list continues to grow, patients with renal failure have longer wait times before an allograft is available. The transplantation of an organ with a cyst or tumor broadens the number of potential grafts available. Formerly, the general practice was to avoid organs with tumors for transplant because of the risk of malignancy transmission to the recipient. However, with comprehensive donor selection and small size of the primary tumor, the positive outcomes of transplant outweigh the risks of transmission after a partial nephrectomy. ${ }^{2}$ Along with living donation and paired donation procedures, expanding the criteria for transplant to include the use of kidneys with masses has provided an opportunity to increase the donor pool.

\section{Case Report}

The donor was a 31-year-old woman, the daughter of the recipient, without significant past medical history. Her ABO blood group was A, and she was positive for both Cytomegalovirus and Epstein-Barr virus. During the workup, she was found to have an 8-mm Bosniac F2 complex cystic lesion in the upper pole of the right kidney (Figure 1). Oncologic urology specialists explained the possible risk of malignancy within that cyst to the patient. After a thorough multidisciplinary discussion between medical personnel from various specialties, including oncology, nephrology, and urology, the ensuing plan was to excise the cyst with healthy margins during the nephrectomy and send the specimen to pathology for 
frozen section analyses before being transplanted to the recipient. The risk for potential transfer of malignancy from donor to recipient was discussed with the recipient (her father), along with the other risks, benefits, alternatives, limitations, and potential complications of the surgery, and an informed consent was obtained.

The recipient was a 69-year-old Hispanic man with a history of ESRD secondary to hypertensive nephrosclerosis and chronic glomerulonephritis. $\mathrm{He}$ received hemodialysis for 3 years before it was discontinued. He had restarted on peritoneal dialysis 4 years later when he was placed on the deceaseddonor kidney transplant list. His $\mathrm{ABO}$ blood group was $\mathrm{A}$, and he was negative for Cytomegalovirus and positive for Epstein-Barr virus. Calculated panelreactive antibody value was $0 \%$, there was $4 / 6$ antigen match, and final crossmatch was negative.

Intraoperatively, the donor's right kidney was removed by hand-assisted laparoscopic right nephrectomy. At the back table, an 8-mm nodule in the kidney was resected and sent to pathology for surgical margin and frozen section analyses (Figure 2). During intraoperative consultation, the pathologist stated that resection margins were negative and that the mass may represent renal cell carcinoma (RCC). The kidney was transplanted to the right lower quadrant. Induction was done with methylprednisolone (500 $\mathrm{mg}$ ) and alemtuzumab (30 mg) subcutaneously. Postoperative immunosuppression was maintained with tacrolimus (1 mg daily) and methylprednisolone (once daily). Permanent sections confirmed that the solid mass was a pT1a chromophobe RCC measuring $1.0 \mathrm{~cm}$ (Figure 3), which was excised with negative margins.

The recipient's postoperative course was uneventful, and he was discharged on postoperative day 7. One week later, the patient presented to the clinic and an ultrasonography revealed a perinephric fluid collection. A percutaneous drain was placed to reduce the collection that turned out to be a lymphocele. At last follow-up, the patient was doing well and his kidney function was normal (creatinine level ranging from 1-1.25 mg/dL). Cancer surveillance with computed tomography imaging showed no evidence of recurrence or metastatic RCC. The prostate-specific antigen levels have remained within normal limits after 38 months of follow-up.

Figure 1. Computed Tomography Scan and Magnetic Resonance Image of Abdomen of Donor
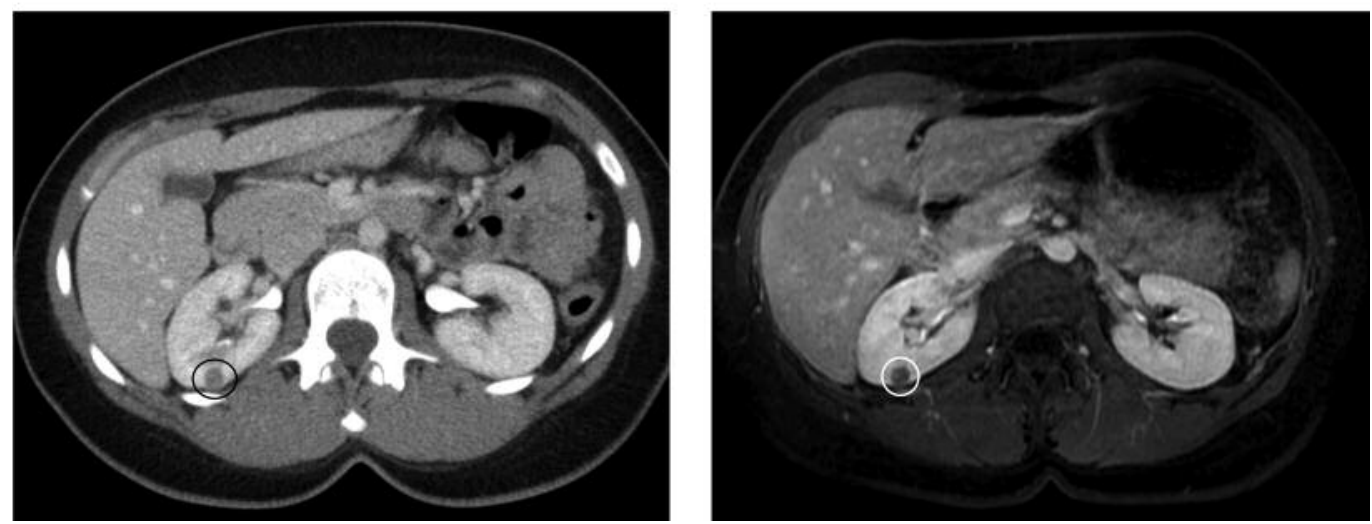

(Left) Computed tomography scan shows an 8-mm mass (black circle) in midpole of the right kidney. (Right) The mass (white circle) can also be visualized via magnetic resonance imaging of the abdomen. After resection of the mass, the kidney was transplanted into the recipient.

Figure 2. At the Back Table, the Renal Tumor Was Excised With 5-mm Margins From the Graft
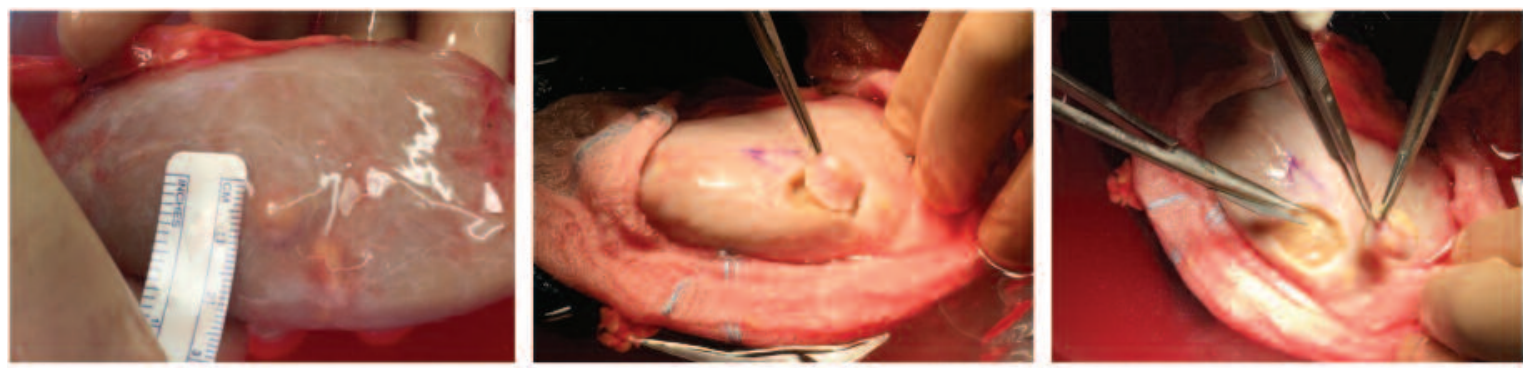
Figure 3. Kidney Histopathology
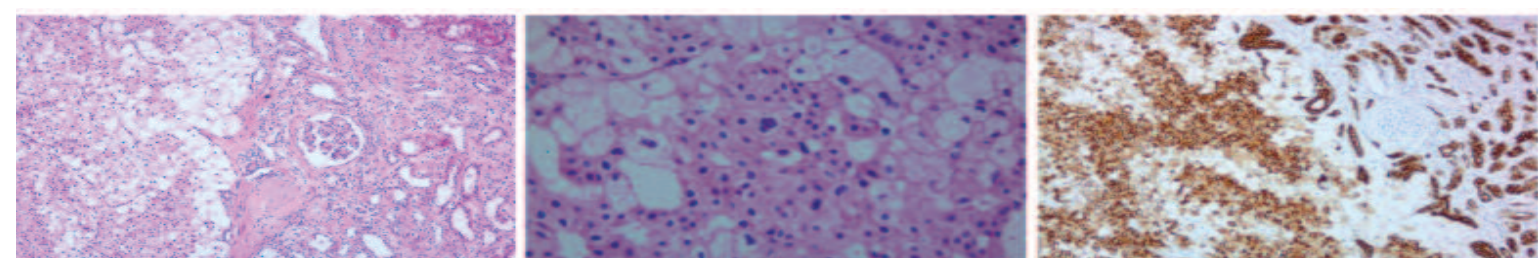

(Left) The tumor is solid and well demarcated from kidney cortical parenchyma (right), with a thin, discontinuous capsule and tubular compression from local mass effect (hematoxylin and eosin, $\times 100$ ). (Middle) Higher magnification (hematoxylin and eosin, $\times 400$ ) of the neoplasm revealed nests of polygonal cells with distinct cell membranes, predominantly granular pale eosinophilic cytoplasm with occasional vacuolization and some foci of cytoplasmic clearing. There was only mild nuclear pleomorphism, rarely evident nucleoli, and perinuclear halos. (Right) Tumor cells were positive on a cytokeratin 7 immunoperoxidase preparation $(\times 100)$, with the granular eosinophilic cells showing the strongest reactivity. The solid growth pattern of the tumor (left) is in contrast to the organized nephrons (right).

\section{Discussion}

Renal transplant remains the optimal treatment option over dialysis for patients with ESRD. There is an increasing discrepancy between patients with ESRD being on the transplant wait list and patients who receive allografts. In the United States, there are an estimated 64000 new cases and 14000 deaths from RCC each year. With expanded criteria for donation, which have increased the permissible age of donation, and the use of better immunosuppressive agents improving the survival of the transplanted organs, more RCCs will incidentally be found in kidney allografts. For patients with small renal tumors, that is, $<3 \mathrm{~cm}$, the growth and metastatic potential of these tumors have been low. ${ }^{3}$ Nephronsparing surgery for these small tumors has been shown to provide excellent patient survival. ${ }^{4}$ Radical nephrectomies may provide great outcomes for recurrence; however, laparoscopic nephrectomy with ex vivo resection of the mass can be more advantageous in both preventing renal malignancy and preserving renal function. ${ }^{5}$

The outcomes of patients undergoing partial nephrectomies or radical nephrectomies for RCCs of $<4 \mathrm{~cm}$ have shown that local recurrence rates are less than $5 \% .{ }^{6} \mathrm{Nicol}$ and associates reported on 43 kidney transplant procedures from patients who consented to transplant after undergoing radical nephrectomies for incidentally detected RCC of $<3 \mathrm{~cm}$ in diameter. Over a mean follow-up of 32 months, there was only 1 recurrence in a 71-year-old patient who was stable under observation. ${ }^{7}$ The study also revealed that recipients of enucleated kidneys had significantly better 5-year survival rates of $88 \%$ versus $74 \%$ for patients who received only dialysis. ${ }^{8}$ In a study from Musquera and associates, 1-year graft survival of $100 \%$ with no tumor recurrence after a mean follow- up of 32.34 months was demonstrated. In that study, 11 transplant procedures from 4 living donors and 4 deceased donors (totaling 8 grafts) showed incidentally small renal masses and 3 contralateral grafts. ${ }^{9}$ Lugo-Baruqui and colleagues identified 4 cases consistent with diagnosis of RCC with stage I TNM classification at the time of transplant. Partial nephrectomies were performed before transplant with no surgical complications. After a median follow-up of 36 months, 3 patients had maintained well-functioning allografts, with 1 noncompliant patient exhibiting renal complications as a result of BK viral infection. All patients, both donors and recipients, reported no recurrence of tumors at the end of follow-up. ${ }^{10}$

In our donor, tumor resection was successful with no development of complications throughout the donor's clinical course. Chromophobe RCCs without necrosis or sarcomatoid morphology have been reported to result in better prognostic characteristics than clear cell and papillary RCCs of similar size and stage; consequently, these are considered low-grade tumors with low metastatic risk. ${ }^{11}$ Currently, both our donor and recipient are cancer free after 30 months of follow-up with the recipient's graft functioning well.

The current suggestion from the ad hoc Disease Transmission Advisory Committee of the Organ Procurement and Transplantation Network/United Network for Organ Sharing is that solitary, welldifferentiated RCCs, Fuhrman nuclear grade I/II tumors of $<1 \mathrm{~cm}$ in diameter have minimal risk of transmission of malignancy or recurrence to the recipient. ${ }^{12}$ Although there are no definitive guidelines for appropriate transplant of renal allografts with incidental findings of malignancies, there are clear benefits in using kidneys after ex vivo partial nephrectomy of the mass. These kidneys 
widen the pool of potential grafts and can reduce the number of patients who die while on the transplant wait list when only receiving dialysis as treatment.

Most authors, including our practice, have utilized calcineurin inhibitors as the immunosuppression protocol of choice. Transplant patients are at an increased risk of developing malignancies such as kidney cancer, nonmelanoma skin cancer, and lymphoma compared with the general population. ${ }^{13}$ Mammalian target of rapamycin (mTOR) inhibitors are an alternative to the known calcineurin inhibitors as they have both immunosuppressive and antioncogenic properties. In a multicenter prospective study (the CONVERT Trial), rates of malignancy in renal allograft patients were compared between patients who received sirolimus and those who received calcineurin inhibitor-free therapies. The Trial showed that there was a lower rate of malignancy after 2 years in patients who converted to sirolimus than in patients who continued on calcineurin inhibitor-based immunosuppression. ${ }^{14}$ With no studies done of specific use of mTOR drugs in kidney allografts resected with RCC, further investigations are needed on this topic before widespread conversion to sirolimus is considered.

\section{Conclusions}

With the increase in number of patients with ESRD, a greater number of possible donors must be considered. For patients with small RCCs, both the growth and metastatic potential of these tumors have been low, and transplant of these kidney grafts has been associated with good graft function and low recurrence rate. Transplant of kidneys from living donors with early-stage RCC can be a great option for patients who have a long wait list time. For these patients, living donations not only extend the life expectancy and quality of life of recipients but also enable early diagnosis and treatment/cure of disease in donors.

\section{References}

1. Saran R, Li Y, Robinson B, et al. US Renal Data System 2015 Annual Data Report: Epidemiology of Kidney Disease in the United States. Am J Kidney Dis. 2016;67(3 Suppl 1):Svii, S1-S305.

2. Frasca GM, D'Errico A, Malvi D, et al. Transplantation of kidneys with tumors. J Nephrol. 2016;29(2):163-168.

3. Chawla SN, Crispen PL, Hanlon AL, et al. The natural history of observed enhancing renal masses: meta-analysis and review of the world literature. J Urol. 2006;175(2):425-431.

4. Flechner SM, Campbell SC. The use of kidneys with small renal tumors for transplantation: who is taking the risk? Am J Transplant. 2012;12(1):48-54.

5. Meng MV, Freise CE, Stoller ML. Laparoscopic nephrectomy, ex vivo excision and autotransplantation for complex renal tumors. J Urol. 2004;172(2):461-464.

6. Russo P. Open partial nephrectomy: an essential operation with an expanding role. Curr Opin Urol. 2007;17(5):309-315.

7. Nicol DL, Preston JM, Wall DR, et al. Kidneys from patients with small renal tumours: a novel source of kidneys for transplantation. BJU Int. 2008;102(2):188-192; discussion 192-183.

8. Brook NR, Gibbons N, Johnson DW, Nicol DL. Outcomes of transplants from patients with small renal tumours, live unrelated donors and dialysis wait-listed patients. Transpl Int. 2010;23(5): 476-483.

9. Musquera M, Perez M, Peri L, et al. Kidneys from donors with incidental renal tumors: should they be considered acceptable option for transplantation? Transplantation. 2013;95(9):1129-1133.

10. Lugo-Baruqui JA, Guerra G, Chen L, et al. Living donor renal transplantation with incidental renal cell carcinoma from donor allograft. Transp/ Int. 2015;28(9):1126-1130.

11. Przybycin CG, Cronin AM, Darvishian F, et al. Chromophobe renal cell carcinoma: a clinicopathologic study of 203 tumors in 200 patients with primary resection at a single institution. Am J Surg Pathol. 2011;35(7):962-970.

12. Nalesnik MA, Woodle ES, Dimaio JM, et al. Donor-transmitted malignancies in organ transplantation: assessment of clinical risk. Am J Transplant. 2011;11(6):1140-1147.

13. Dantal J, Pohanka E. Malignancies in renal transplantation: an unmet medical need. Nephrol Dial Transplant. 2007;22 Suppl 1:i4i10.

14. Alberu J, Pascoe MD, Campistol JM, et al. Lower malignancy rates in renal allograft recipients converted to sirolimus-based calcineurin inhibitor-free immunotherapy: 24-month results from the CONVERT trial. Transplantation. 2011;92(3):303-310. 